\title{
Astronomical Object Index
}

\begin{tabular}{|c|c|c|}
\hline $\mathrm{BD}+30363981$ & IRC+10216 179, 184, & NGC 253162 \\
\hline & $185,186,192,194$ & NGC 628138 \\
\hline 396 (G39.2-0.3) 173 & 195-198, 236, 298, & NGC 891161 \\
\hline $\begin{array}{l}\text { Ced } 201224 \\
\text { Ced } 1730 \text { (G39.2-0.3) }\end{array}$ & 362 & NGC 1068385 \\
\hline Chi Cygni 186 & IRS 48281 & NGC 1569163 \\
\hline Cloverleaf galaxies 7,8 & & NGC 2023 20, 210 \\
\hline CRL 618 235, 237, 242 & Jupiter 236 & NGC 2974164 \\
\hline CRL 2688382 & & NGC 3962164 \\
\hline & Kes 17 (G304.6+0.1) & $\begin{array}{l}\text { NGC } 453615 \\
\text { NGC } 4589165,166\end{array}$ \\
\hline D7 Cru 409, 410 & 173,174 & NGC 519436 \\
\hline DM Tau 281 & Kes 69 (G21.8-0.6) & NGC 519535,36 \\
\hline 30 Dor 400, 401, 418 & & $\begin{array}{l}\text { NGC } 5529161 \\
\text { NGC } 571336\end{array}$ \\
\hline $\begin{array}{l}\text { Galactic Center } 384 \text {, } \\
\quad 385\end{array}$ & $\begin{array}{l}\text { Large Magellanic } \\
\text { Cloud 17, 20, 48, } \\
\text { 158, 170, 173, } \\
236,351,418\end{array}$ & $\begin{array}{l}\text { NGC } 586636 \\
\text { NGC } 5907161 \\
\text { NGC } 694636 \\
\text { NGC } 702350,51,85,\end{array}$ \\
\hline HD 10412273 & Lick H $\alpha 234$ 173, 174 & $210-213,224,225$ \\
\hline HD 14154276 & & $228,230,233,401$ \\
\hline HD 3428252 & M17 18, 58, 59, 400, & 403,450 \\
\hline HD 95881273 & 404 & NGC 7027 4, 6,15, 20, \\
\hline HD 97048262,263 , & M31 58, 351 & 81,382 \\
\hline 281 & M33 351 & NGC $733136,173,174$ \\
\hline HD 141569281 & M81 163 & \\
\hline HD 163296281 & M82 $17,35,52,53$, & Orion Bar $6,15,18$, \\
\hline HD 169142281 & $161,162,163$ & $20,58,127,128$ \\
\hline 52 & $\begin{array}{l}\text { 2MASX } \\
\quad \mathrm{J} 16205879+5425127\end{array}$ & 400,404 \\
\hline IRAS 11308173 , & 152 & $\mathrm{P} /$ Encke 322 \\
\hline 174 & Magellanic clouds 29, & P/IRAS 322 \\
\hline IRAS $08572+3915$ & $30,31,40,137,162$, & Puppies A supernova \\
\hline 385 & 167, 216ff, 224, 236, & remnant 174,175 \\
\hline IRAS 13418-6243 120, & 351,403 & \\
\hline 121 & Milky Way 29, 30 & QV Vul 408, 409, 411 \\
\hline IRAS $23133+6050120$ & & \\
\hline 12 & N132D 170, 173 & RCW 29 160, 161 \\
\hline
\end{tabular}


Red Rectangle 120, 121, SMP LMC 11236 126, 365

Rho Oph 224

RW Leo, see IRC+10216

Titan 236, 242, 247

Tycho supernova

remnant 159

Saturn 236

Serpens molecular cloud 260

Small Magellanic Cloud 137, 215ff, 351
V2361 Cyg 409

V2362 Cyg 409, 410

V Cygni 186

Vela supernova remnant 160

VII Zw 353152

V705 Cas 408, 409, 411

V842 Cen 408, 409, 411

V854 Cen 179, 186, 187
W33A 255

Wild-2 322 